\title{
Experimental Analysis of UWB Signal Performance in a Constrained Environment for Railway Application
}

\author{
Ouafae Cohin ${ }^{1}$, Sara Ibenjellal1,2, Fouzia Boukour ${ }^{3}$, Sylvie Baranowski² \\ ${ }^{1}$ ICAM, Lille, France \\ ${ }^{2}$ IEMN, Université de Lille, Lille, France \\ ${ }^{3}$ IFSTTAR, LEOST, Villeneuve d'Ascq, France \\ Email: cohin@yahoo.fr
}

Received 29 July 2015; accepted 9 October 2015; published 12 October 2015

Copyright (C) 2015 by authors and Scientific Research Publishing Inc.

This work is licensed under the Creative Commons Attribution International License (CC BY).

http://creativecommons.org/licenses/by/4.0/

(c) (i) Open Access

\begin{abstract}
In the railway application, in order to establish the communication between wagons, some technologies are proposed such as the ZigBee and HTN (Hybrid Networking Technology). However, these techniques have some limitations such as: the low data rate, non-secured transmission and interferences. The Ultra Wide Band (UWB) technology presents a good alternative and a good candidate for this application. In recent years, UWB communication systems have received significant attention from both the industry and the academia. In February 2002, the Federal Communications Commission (FCC) allocated $7500 \mathrm{MHz}$ of spectrum (from $3.1 \mathrm{GHz}$ to $10.6 \mathrm{GHz}$ ) for use by UWB devices. This ruling has helped to create new standardization efforts, like IEEE 802.15.3a, which focus on developing high speed wireless communication systems. The application of the UWB radio technique in the transport is a more recent topic that is thoroughly researched considering several factors including the nature of the transport propagation environment; the use of adequate transmitting/receiving duty cycle (LDC); the number of devices using UWB technology, the types and levels of interferences. Regulation bodies have considered these railway applications. Impulse Radio Ultra Wideband (IR-UWB) systems have been studied for their inherent advantages of coexistence with narrowband systems with high data rate over short distances with sufficiently small amount of transmitted power. The UWB systems are highly susceptible to interference between the coexisting narrowband systems because of very low transmission power. This paper considers the use of the UWB radio technology for railway application. In this paper, we evaluate the communication performance in constrained environment for the railway application.
\end{abstract}

\section{Keywords}

UWB, Channel Model, Channel Simulation, Simulation Analysis 


\section{Introduction}

Railroads play an important role in our society today by transporting people and heavy freight. Indeed, the demand for railway services is consistently increasing; stricter safety requirements for railway signaling, control and infrastructure are needed. Accompanying that trend, currently, the railway companies must be adequately ensured to cover their liabilities in traceability and wagons localization, verification of presence of the wagon and the integrity of train, monitoring of freight trains, and also in automation of coupling between wagons,

This paper concentrates on the communication issue. It starts by an evaluation of the performance of such UWB signals propagating between two wagons; the concept is shown in Figure 1. Analysis and implementation of wagon communication systems based on UWB require an accurate channel to determine that can be achieved. In our case of application, and for a better adaptation of the model, we have compared the IEEE 802.15.3 astatic channels models to measurements [1]-[3].

To deploy wireless networks on the train, a study of the propagation of electromagnetic (EM) waves is required in this particular environment. Indeed, the wireless communications within and between wagons can be severely limited by the metallic structure of their walls. In this respect, it is fundamental to ensure the coexistence between different systems.

Ultra-Wideband (UWB) pulses have extremely short time widths of less than 1 ns and extremely broad bandwidths of more than $1 \mathrm{GHz}$. Systems using UWB pulses are usually considered to have high capacity and be robust in multi-path Environments [4]. UWB employs base band pulses of very short duration. The very low energy density results in low probabilities of intercept and detection. The UWB advantages in high speed wireless communication are due to absence or minimal multipath effects. The inherent high range resolution provides accurate measurement of distance, which permits geolocations to accuracies of few centimeters. The interference robustness of UWB communications comes through its ability to eliminate the effects of multi-path fading. Normally, a communications system needs to have an additional margin of power to maintain the planned quality of service in the presence of multi-path fading.

This paper is organized as follows: the second part presents the UWB technology and the propagation channel modeling using the IEEE 802.15.3a channel models. The third section provides the measurement results of the UWB system in free and with the metallic constraints. The fourth section provides a comparative study between experimental and simulation results of the UWB system in terms of constraints. The fifth section concludes the paper and gives some perspectives.

\section{The Proposed UWB Technology}

\subsection{Definition}

Ultra-wideband (UWB) technology offers a promising solution to the RF spectrum drought by allowing new services to coexist with current radio systems with minimal or no interference. This coexistence brings the advantage of avoiding the expensive spectrum licensing fees that providers.

\subsection{Simulation}

The structure of the channel model has a strong influence on the system performance assessment. The 802.15 channel model is based on some of the measurement campaigns published in the open literature see, e.g., [5]-[7], as well as on measurement campaigns performed explicitly for the standard. These new campaigns were carried out by various standardization participants, and their data were used to assess the goodness of fit of various proposed channel models, as well as to calibrate their parameters.

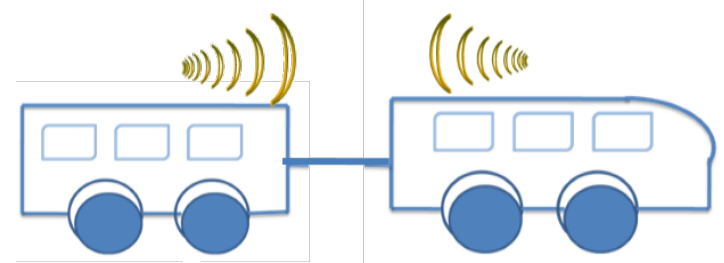

Figure 1. Wireless communication concept between wagons. 
In the literature, four different measurement environments were defined, namely CM1, CM2, CM3, and CM4. CM1 describes a LOS (line-of-sight) scenario with a separation between transmitter and receiver of less than 4 $\mathrm{m}$. CM2 describes the same range, but for a non-LOS situation. CM3 describes a non-LOS scenario for distances between TX and RX 4 - $10 \mathrm{~m}$. Scenario 4 finally describes an environment with strong delay dispersion, resulting in a delay spread of 25 ns. Note that, when using the model, the total average received power of the multipath realizations is typically normalized to unity in order to provide a fair comparison with other wideband and narrowband systems.

This study has been established in the perfect synchronization case between the transmitter and the receiver. The receiver and the transmitter block diagrams for UWB communication system are depicted in Figure 2. We simulated the system UWB based on BPSK (Binary phase shift keying). This modulation is based on the information coding by the pulse polarity. (Positive polarity if the bit is 0 or negative, for example if the bit is 1 ). The position of the pulse remains fixed, and it is only the phase varies, there by simplifying synchronization Figure 3. The modulation with our receiver system has been done with channel AWGN and using the IEEE 802.15a channels, in order to evaluate its performances in terms of data rate and Bit Error Rate (BER) [8].

UWB impulses are generally narrow time pulses of sub-nanosecond or picoseconds order. The amplitude of the pulse should be normalized to comply with the above-discussed FCC mask [5] [7]. We have used the Gaussian pulse waveform. Figure 3 illustrates the principle of coding and the emitted signal.

After spreading, the signal is modulated by a binary phase-shift keying (BPSK modulation) and then an additive white Gaussian noise (AWGN) was integrated. BPSK has an advantage over pulse amplitude and position modulation due to an inherent 3-dB increase in separation between constellation points. The performance of the

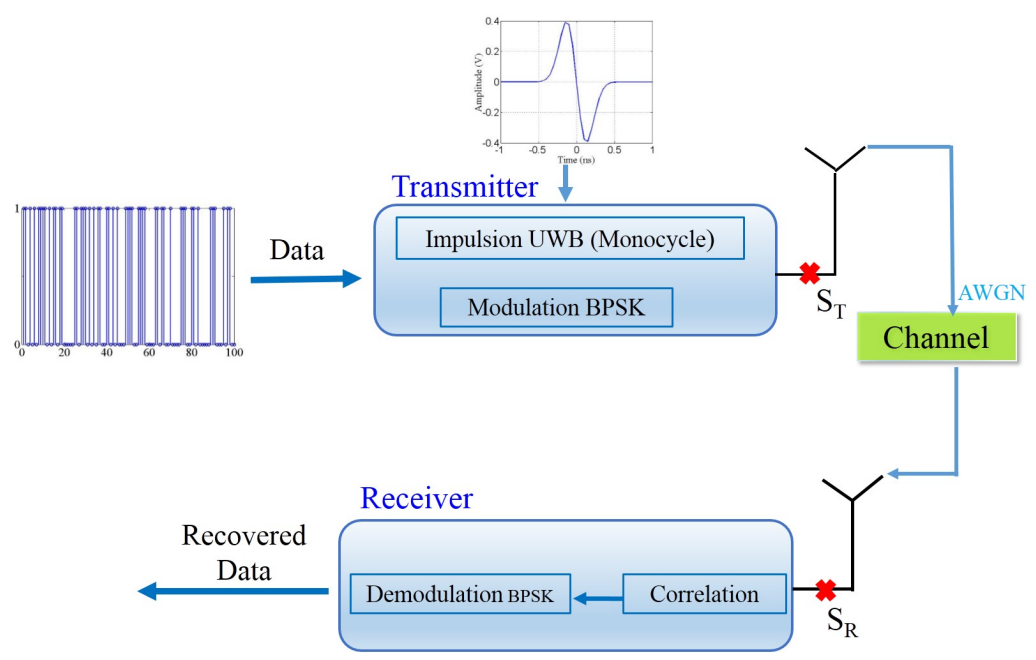

Figure 2. Diagram of the UWB channel simulation.

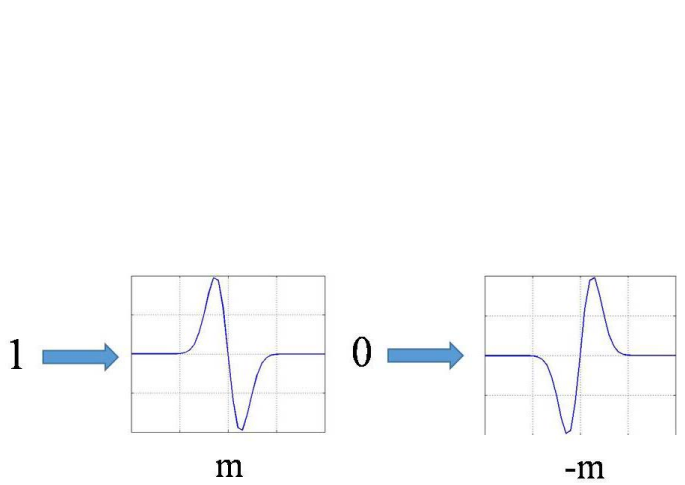

(a)

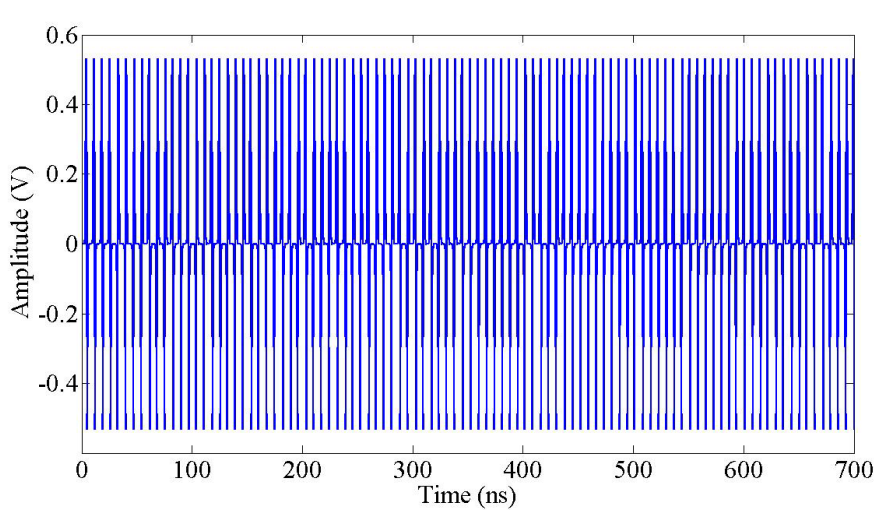

(b)

Figure 3. (a) Principle of coding; (b) Emitted signal (ST). 
communication has been evaluated in the presence of additive white Gaussian noise for the simulation channel of Figure 2 and for the four 802.15 channel models. The BER simulation results under the different channels are given in Figure 4. The SNR (signal-to-noise ratio) is defined by $E_{b} / N_{o}$, where $E_{b}$ is the energy per user bit and $\mathrm{N}_{\mathrm{o}}$ is the single-sided noise power spectral density.

We notice that the BER decreases in an exponential way when the SNR increases for the first channel proposed in Figure 2. The BER performance of different models present a high degradation due to different environmental constraints taken into account as there flections, diffraction, multipath and dispersion. The 802.15 standard model is an important step for the understanding of UWB channels and the environment effect on the performance of communication. It is the purpose of the remainder of this paper.

\section{Experimental Description}

\subsection{Description of the Experimental Setup}

In this part we describe the experimental setup. The test bench consists in an Arbitrary Waveform GeneratorAWG7102 connected to a Digital Storage Oscilloscope (TDS6124C) with a 4 meters long cable. This study has been established in the perfect synchronization case between the transmitter and the receiver. The transmission and reception of signals are performed by two antennas SAS -571 Double Ridge Guide Horn Antenna which the Broad Frequency Range is of $700 \mathrm{MHz}$ to $18 \mathrm{GHz}$. This type of antenna is lightweight, compact and has been manufactured for maximum gain, low VSWR and broadband response [9].

The transmission and reception of signals are performed by two antennas DRG HORN (emv). All measurements have been performed at fixed height, $1.45 \mathrm{~m}$ in a free space. As described in the following Figure 5.

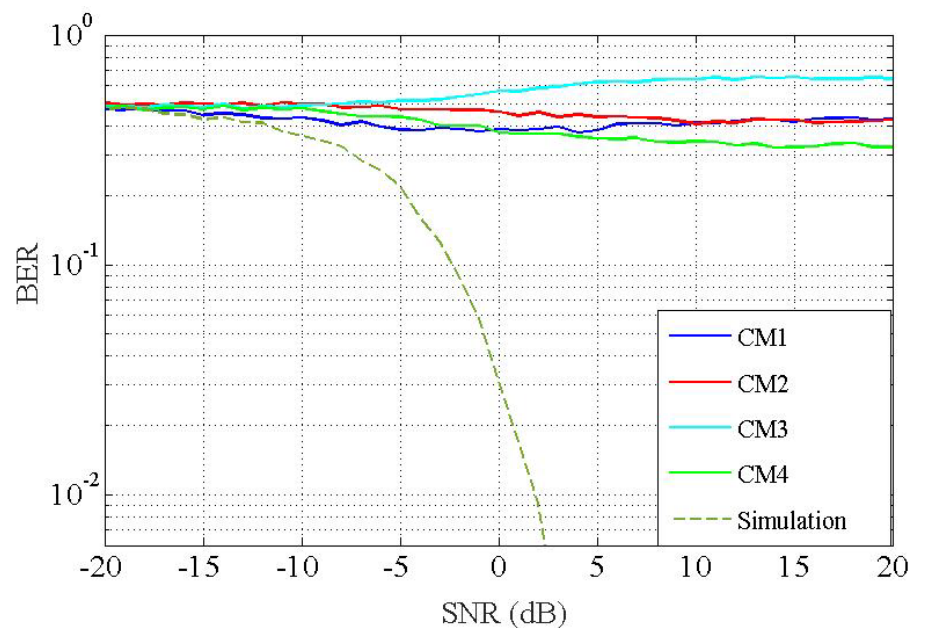

Figure 4. Simulations of the BER performance.

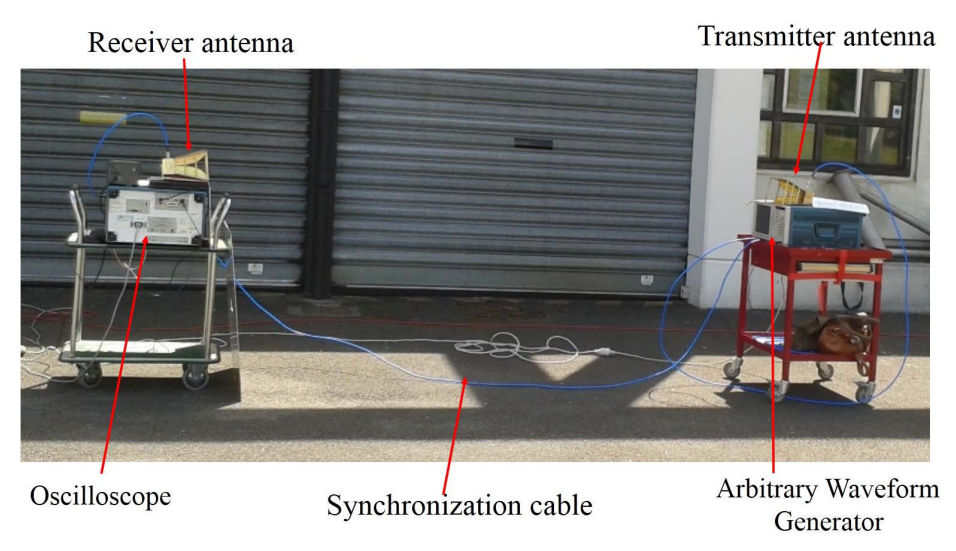

Figure 5. Top view of the measurement setup. 


\subsection{Measurement: Free Space}

In this section we measure the received signal $S_{R}$ in free space. The comparison with the emitted signal $S_{T}$ is shown in Figure 6. The information (data) is carried by the amplitude of the pulses. We can note that the received signal (blue curve) decreased significantly because of the distance attenuation and the environment noise. In narrowband signals with a very narrow frequency band, the change in frequency only minimally changes the received power and hence can be overlooked.

However, due to the wide range of frequencies covered by the UWB spectrum, the received power drastically changes and thus distorts the pulse shape. This will limit the performance of UWB receivers that correlate the received pulses with a predefined template such as classical matched filters.

\subsection{Measurement: Evaluation of the Metallic Surface}

The quality of a radio link between two nodes is associated with: equipment, modulation, interferences and the propagation medium. Even though some data are not accessible, the interferences and the propagation channel of the environment often have significant impact on the transmission.

In this section, we compare the measurement results in two cases using IEEE 802.15.3a channel models.

- The first case: In free space without metallic plate, Line Of Sight (LOS).

- The second case: In free space with metallic plate, No Line Of Sight (NLOS).

Thereafter, we study the effect of the metal on propagation channel. To assess the impact of the metal walls of the train on the signal, the metallic plates have been added in front of the transmitter and receiver block as seen in Figure 7. The straight line between a transmitter and a receiver is the line of sight (LOS); the reflected signals from surfaces are non-line of sight (NLOS) [10].

As shown in Figure 8, the received signal with metallic plate has been degraded, the phenomenon is unavoidable in wireless communications channels. It is caused by multiple reflections of the transmitted signal from metallic surfaces added. In a UWB communication system, the signal arriving at the receiver antenna consists of many multipath components, each of which is the result of the interaction of the transmitted rays with the surrounding objects or environments.

The effect of multipath is rather severe for narrow band signals; the signal degradation is up to $-40 \mathrm{~dB}$ due to the out-of-phase addition of LOS and NLOS continuous waveforms. On the other hand, the very short duration

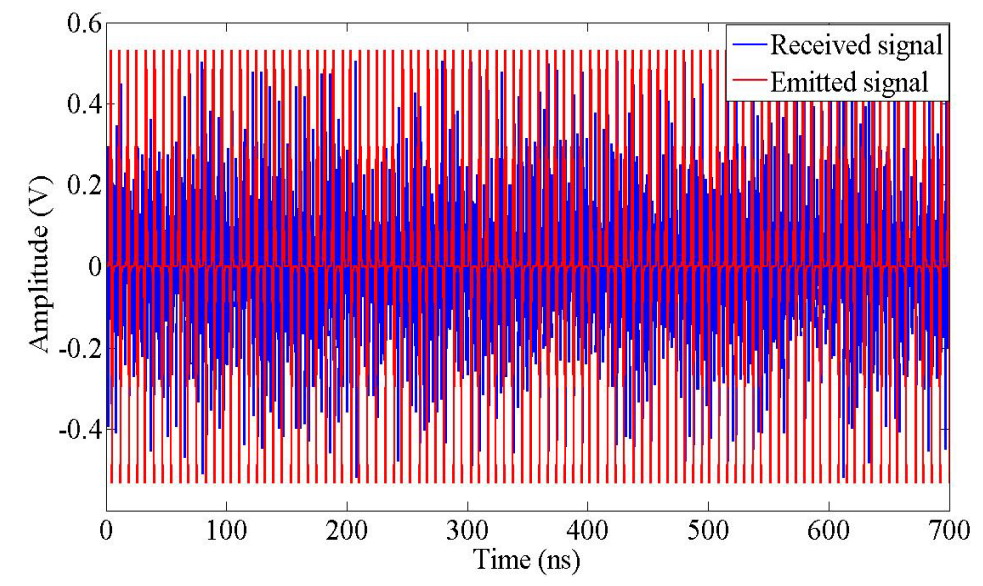

Figure 6. Comparison between emitted and received signal.

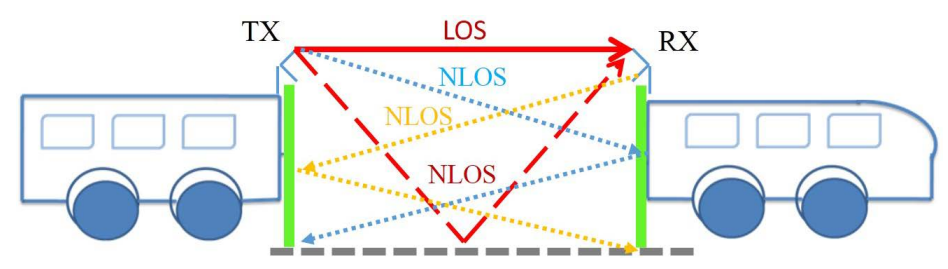

Figure 7. The multipath phenomenon in wireless links. 
of UWB pulses makes them less sensitive to the multipath effect. Because the transmission duration of a UWB pulse is shorter than a nanosecond in most cases, the reflected pulse has an extremely short window of opportunity to collide with the LOS pulse and cause signal degradation. Although the short duration of UWB pulses makes them less sensitive to multipath effects compared to narrowband signals, it doesn't mean that UWB communications is totally immune to multipath distortion (Figure 9).

\subsection{BER Comparison}

In order to evaluate the performance of the UWB communication with the metallic plates, we calculate the BER according to the SNR of the real propagation channel. For this, we have added the AWGN to the received signal $\mathrm{S}_{\mathrm{R}}$ as shown in Figure 10.

In this paper, Case CM2 and CM4 (4 - 10 m, NLOS) condition of the channel have been used. Then we have compared the calculated BER with de simulated one (Figure 10) and with CM2 and CM4. Figure 11 shows the comparison between BER of measurement with added noise and the simulated BER [11]. The performance of

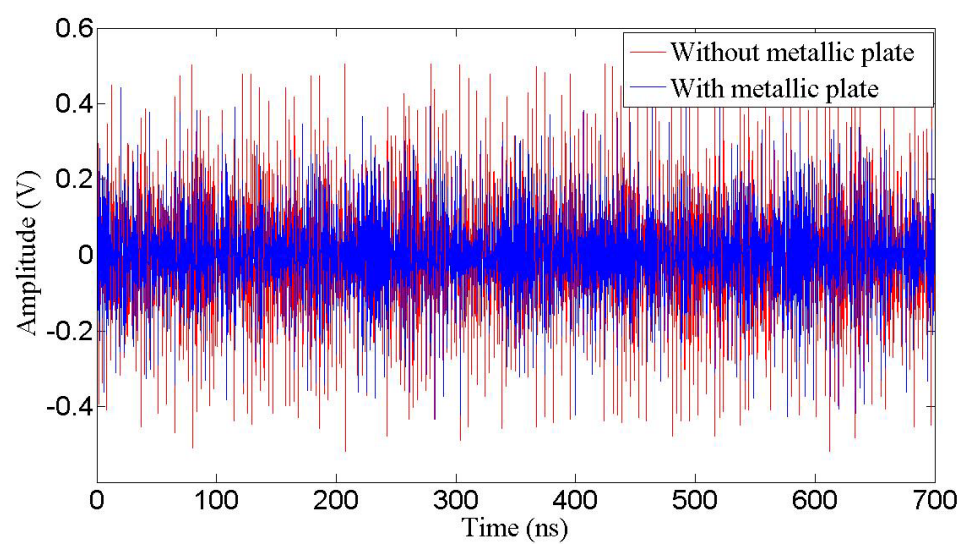

Figure 8. Received signal: evaluation of the metallic plate effect.

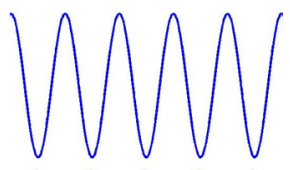

LOS

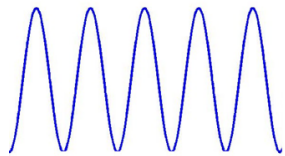

NLOS

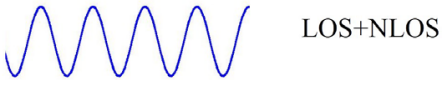

Figure 9. Multipath’s effects on narrowband signals.

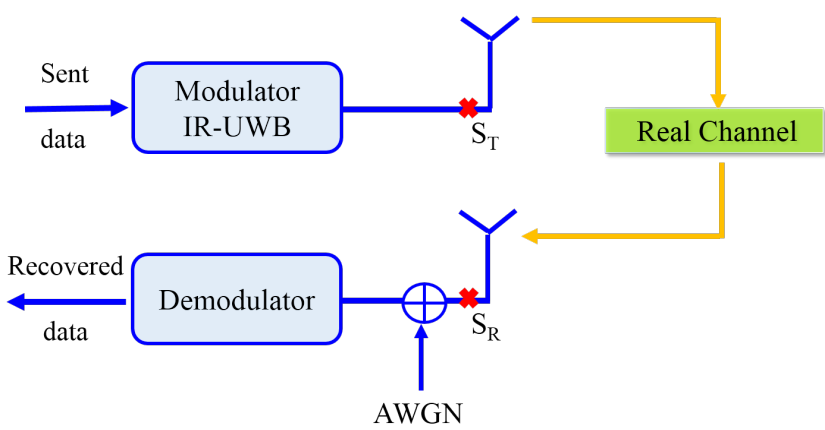

Figure 10. Diagram of UWB system. 


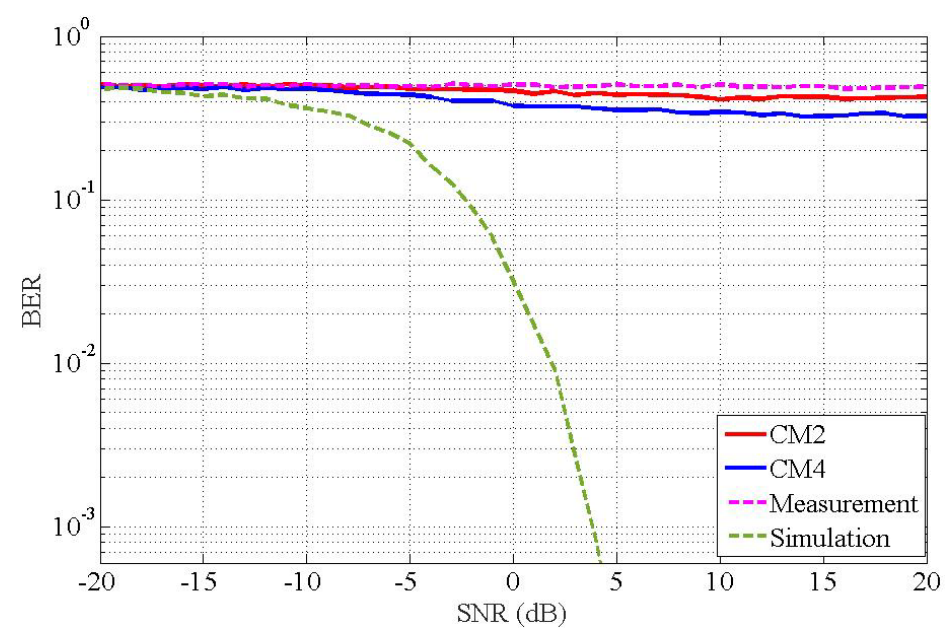

Figure 11. Comparison of the BER performance.

each case has been evaluated. For this purpose, the BER has been computed with Matlab for different SNR values, using the BPSK demodulation [12] [13].

The green curve is obtained by simulation as defined in paragraph 2.2. The BER of the pink curve was obtained from the received signal measured with metallic plate, and after having added channel noise (AWGN) [14]. The red and blue curves represent the BER of CM2andCM4modelsrespectively. We can show significant degradation BER performance (pink curve) when we add the metallic plate, this is certainly due to the errors of experiment and the increase of the number of multipath. The results show also a good agreement between BER performance over IEEE.15.3a models and measurement channel.

\section{Conclusions}

This paper provides a first experimental approach to analyzing UWB performance in a constrained environment with metallic walls. UWB transmission systems have several advantages like high data rate, robust to multipath fading, potential low-cost transceiver implementations, accurate mobile user location, and coexistence with narrowband wireless systems. However, development of UWB technology in specific environments like railway application to live up to its full potential still poses significant technical challenges.

The BER performance of the UWB system is compared in different cases to evaluate the effect of the metallic walls. This study proves that the approach based on the experimentations shows the metallic constraints effect on the communication by performing the received signal, the recovered data and the BER. This step gives us an idea about the effect of the metallic walls on the communication performance, but these experimentations are not sufficient to characterize and predict in railway environment. To solve this problem, measurements in real environment are needed. In the future work, the tests will be realized in real railway propagation environments.

\section{References}

[1] European Telecommunications Standards Institute (ETSI) (2005/2006) Electromagnetic Compatibility and Radio Spectrum Matter (ERM); Short Range Devices (SRD); Ground- and Wall-Probing Radar Applications; Part 1 and Part 2. Technical Report ETSI EN 302 066-1 V1.1.1.

[2] (2004) Federal Communications Commission Rules, Subpart F Ultra-Wideband Operation, CFR47, Chapter I, 15.

[3] (2006) Radio Frequency Devices, FCC Part 15, Feb. 16.

[4] Dilmaghani, R.S., Ghavami, M., Allen, B. and Aghvami, H. (2003) Novel UWB Pulse Shaping Using Prolate Spheroidal Wave Functions. Proceedings of 14th IEEE International Symposium on Personal, Indoor \& Mobile Radio Communications (PIMRC2003), Beijing, September, 602-606.

[5] Benedetto, M.-G. and Vojcic, B.R. (2003) Ultra Wide Band (UWB) Wireless Communications: A Tutorial. Journal of Communication and Networks, Special Issue on Ultra-Wideband Communications, 5, 290-302.

[6] El Abed, A., Boukour, F., Elhillali, Y. and Rivenq, A. (2009) V2V Communication System Using Orthogonal Functions and Bipolar PPM Coding. Proceedings of ITST Conference, Lille, 20-22 October 2009. 
[7] Elbahhar, F., Rivenq, A., Rouvaen, J.M., Heddebaut, M. and Boukour, T. (2005) Comparison between DS-CDMA and Modified Gegenbauer Functions MGF for a Multi-User Communication Ultra Wide Band System. IEE Proceedings Communications, 22 April 2005.

[8] Elbahhar, F. (2004) Multi-User Ultra Wide Band Communication System Based on Modified Gegenbauer and Hermite Functions. PhD. Thesis, University of Valenciennes, Valenciennes.

[9] http://www.ahsystems.com/datasheets/SAS-571_Horn_Antenna_Datasheet.pdf

[10] Robins, K.W. (1972) Short Baseband Pulse Receiver. US Patent 3,662,316.

[11] Khan, M.G., Nordberg, J., Mohammed, A. and Claesson, I. (2006) Performance Evaluation of Rake Receiver for UWB Systems Using Measured Channel Industrial Environments. International Conference on Wireless Broadband on Ultra Wideband Systems, March 2006.

[12] Maatougui, L., Hamidoun, K., El Hillali, Y., Rivenq, A., Hajjaji, A. and Ouahmane, H. (2015) Intelligent Receiver for M-OAM IR-UWB Modulations System. International Conference on Advanced Logistics and Transport (ICALT), Valenciennes, 20-22 May 2015, 111-115. http://dx.doi.org/10.1109/icadlt.2015.7136604

[13] Elbahhar, F., Rivenq-Menhaj, A. and Rouvaen, J.M. (2005) Multi-User Ultra Wide Band Communication System Based on Modified Gegenbauer and Hermite Functions. Wireless Personal Communications, 34, 255-277. http://dx.doi.org/10.1007/s11277-005-3922-2

[14] Zhuang, W., Shen, X. and Bi, Q. (2003) Ultra Wideband Wireless Communications. Wireless Communications and Mobile Computing, 3, 663-685. http://dx.doi.org/10.1002/wcm.149 